\title{
Charge Measurement of Cosmic Ray Nuclei with the PSD of DAMPE
}

\section{Tiekuang Dong* ${ }^{*}$, Yapeng Zhang ${ }^{2}$, Pengxiong $\mathrm{Ma}^{1}$, Paolo Bernardini ${ }^{3}$, on behalf of the DAMPE Collaboration ${ }^{\dagger}$ \\ $\ddagger$}

${ }^{1}$ Key Laboratory of Dark Matter and Space Astronomy, Purple Mountain Observatory, Chinese Academy of Sciences, Nanjing 210034, China

${ }^{2}$ Institute of Modern Physics, Chinese Academy of Sciences, 509 Nanchang Road, Lanzhou 730000, China

${ }^{3}$ Istituto Nazionale di Fisica Nucleare (INFN) - Sezione di Lecce, I-73100 Lecce, Italy

E-mail: tkdong@pmo.ac.cn

The Plastic Scintillator Detector (PSD) on top of DAMPE can be used to identify the species of cosmic ray particles by measuring their charge. In this paper, the procedure of charge measurement by PSD is described, including alignment of PSD, light attenuation correction, quenching and equalization correction. After these corrections we can get high precision charge spectrum, from which almost all the elements in cosmic rays from $\mathrm{H}$ to $\mathrm{Ni}$ can be clearly identified.

36th International Cosmic Ray Conference -ICRC2019-

July 24th - August 1st, 2019

Madison, WI, U.S.A.

\footnotetext{
* Speaker.

${ }^{\dagger}$ for collaboration list see PoS(ICRC2019)1177

$\ddagger$ This work is supported by National Key Program for Research and Development (No. 2016YFA0400200), and by the National Natural Science Foundation of China (Grant Nos. U1738205, 11673075, 11643011, 11673047, U1738127, U1631111, and 11761161001), and by the Strategic Priority Research Program of the Chinese Academy of Sciences (No. XDB23040000). In Europe the work is supported by the Italian National Institute for Nuclear Physics, and the Italian University and Research Ministry.
} 


\section{Introduction}

DArk Matter Particle Explorer (DAMPE) is a space-based mission [1]. The main scientific objectives of DAMPE include: (1) to search for signals from dark matter decay and/or annihilation by detecting electron/positron spectrum [2], (2) to investigate the origin and propagation of cosmic rays by measuring the spectra of cosmic ray nuclei up to several tens of $\mathrm{TeV}$ or beyond, (3) to detect gamma rays in the Galaxy. The detailed description of DAMPE can be found in the mission paper [3]., while here we only give a brief introduction of DAMPE for the convenience of following discussions. Fig.1 shows the structure of DAMPE. It is composed of four sub-detectors: the Plastic Scintillator Detector (PSD), the Silicon-Tungsten tracKer-converter (STK), the BGO imaging calorimeter (BGO), and the NeUtron Detector (NUD).

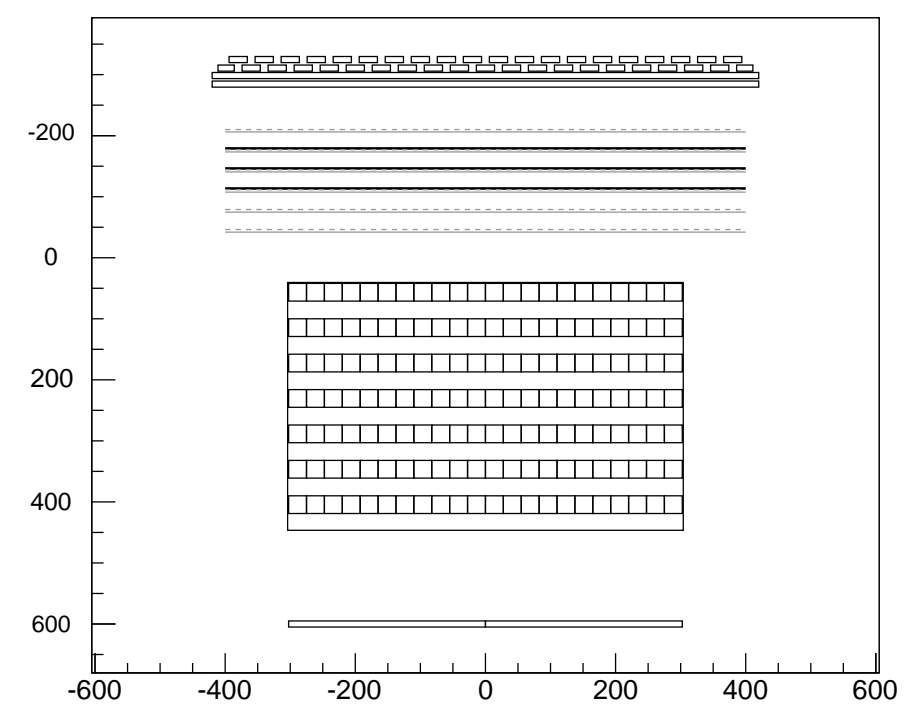

Figure 1: Diagram of DAMPE and the satellite.

\section{Charge Reconstruction Method}

The detailed structure of the Plastic Scintillator Detector (PSD) can be found in Ref.[4]. Here we just give a brief description. The PSD is composed of two planes: the upper/lower plane is arranged along the $\mathrm{X} / \mathrm{Y}$ axis and $41 \mathrm{PSD}$ bars are placed in each plane in the same direction with a two-layer pattern. The size of a PSD bar is $884 \mathrm{~mm}$ long $\times 28 \mathrm{~mm}$ width ( $25 \mathrm{~mm}$ for bars at edges) $\times 10 \mathrm{~mm}$ thickness. In the same plane the neighbouring bars are staggered by $8 \mathrm{~mm}$ to ensure a full active area of scintillators. With this crisscross structure, an incident particle would penetrate at least two and at most four bars. This arrangement can improve the detection efficiency and accuracy of charge measurement. Each PSD bar is viewed by two photomultiplier tubes (PMTs) at the ends.

The basic idea of charge measurement by the PSD is that the energy released by a penetrating charged particle in passing through a material (the plastic scintillator, in this case) is proportional 
to the square of its electric charge. According to the geometrical structure shown above, the PSD is regarded as a 4-layer detector, each scintillating bar (with two PMTs) providing a double measurement of a single crossing particle. So, for an incident particle we can get at least 4 and at most 8 charge measurements. Finally, the arithmetic mean value is calculated as the best estimation of the charge. Based on these ideas, the following 4 corrections should be made:

(1) alignment correction,

(2) light attenuation correction,

(3) equalization correction,

(4) quenching correction.

More information and details can be found in our recent paper [5].

\subsection{Track selection}

Two sub-detectors can measure the track of an incident particle: the STK and the BGO. STK is a thin detector with 6 pairs of silicon strip layers [2]. Each layer can measure a pair of coordinates $(x, y)$. Through the Kalman filter algorithm we can reconstruct the tracks of charged particles including the primary particle and secondary particles. BGO is a thick detector with 7 pairs of BGO layers. The main axis of the shower profile can be used to estimate the track of the incident particle. However, MC simulation shows that the BGO track usually deviates from the real track quite largely with respect to the required accuracy of charge reconstruction. So, the STK track will be used. However, according to the hadronic reaction theory many charged secondary particles are pions $\left(\pi^{ \pm}\right)$and it is difficult to distinguish the primary proton and secondary $\pi^{ \pm}$using only STK signals. For such reason, all events are divided in two classes: heavy nuclei candidates and light nuclei ones. If both the maximum energy deposition in the first and second layer of PSD bars are larger than $20 \mathrm{MeV}$, the event is labeled as a heavy nucleus candidate. Otherwise, the event is labeled as a light nucleus candidate. For a heavy nucleus candidate, the STK track with the largest mean energy deposition is selected as the real track. While for a light nucleus candidate, the STK track which is the nearest to the BGO track is selected. Furthermore, a loose homogenization condition is set: the maximum energy deposition must be less than 5 times of the minimum one along the selected track.

\subsection{Alignment correction}

For a heavy charged particle, the total ionization energy loss in the detector unit is proportional to the path length. For a given path length, the energy deposition is proportional to the square of the charge number. Since the cosmic rays hit the detector from all directions, the real path length must be determined at first using the reconstructed track, then the energy deposition per centimeter is calculated. However, the real geometrical parameters of the PSD bars slightly deviate from the designed values due to several factors, such as limited installation precision, vibration during launching. So, it is necessary to calibrate the real geometry of all PSD bars using proton minimum ionizing particle (MIP) events. The detailed methods of alignment can be found in Refs.[6, 7]. Actually, it is found that, before the alignment correction, two peaks are present in the charge spectrum for proton MIP events, while after the alignment correction, only one peak with higher resolution survives. 


\subsection{Light attenuation correction}

In order to achieve the charge measurement with both PMTs of a single bar, we must determine the impact position of the incident particle. It is because the scintillating light excited by charged particles at a point will be partially absorbed until be collected by a PMT. The attenuation level depends on the distance between the impact point and the PMT, so the charge measured by a PMT, which is proportional to the collected light, also depends on the impact position. According to the principle of light absorption in matter, the light attenuation is expected to obey the exponential decay function. In fact, we find that the attenuation behavior only roughly can be described by an exponential decay function (Fig.2). The formula used in this paper is the sum of an exponential decay function and a cubic polynomial function:

$$
Q_{M P V}(d)=C_{0} e^{-d / \lambda}+C_{1} d+C_{2} d^{2}+C_{3} d^{3}
$$

where $d$ is the distance between the impact point and the PMT, $Q_{M P V}$ is the MPV value of charge spectrum for proton MIP events.

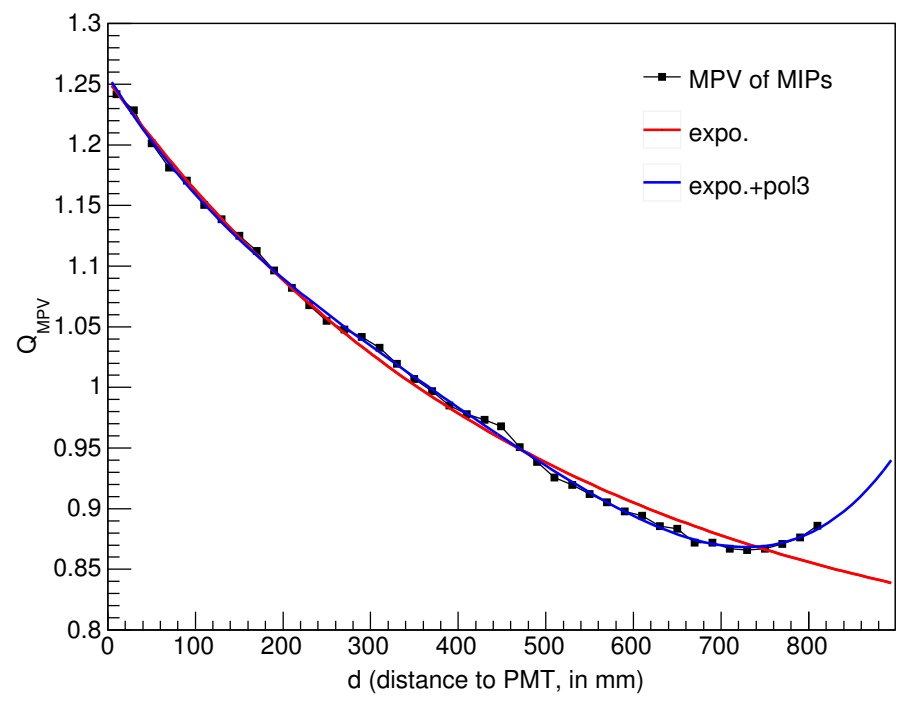

Figure 2: The MPV value of charge spectrum for proton MIP events versus the distance between the impact point and PMT.

\subsection{Equalization correction}

Although produced under the same standard, the PSD bars and PMTs are not perfectly identical one to each other. This means that the peak positions of a charge spectrum measured by different PMTs will deviate from each other. In other words, in measuring the charge value of a certain element, this will broaden the charge spectrum (i.e. will reduce the resolution) when the measurements by all PMTs are put together. In order to improve the charge resolution, the peak positions of the charge spectra measured by all PMTs are imposed to overlap one each other. 


\subsection{Quenching correction}

The last main correction arises from the quenching effect. When nuclei with low charge numbers pass through the PSD, the yield of scintillating light is proportional to the energy deposition. Instead, when the charge value increases, the yield of scintillating light is no longer proportional to the energy deposition. The quenching effect becomes more and more severe with the increase of the charge number. For example, before quenching correction the peak position of $\mathrm{C}(Z=6)$ is about 5.9, but the peak position of $\mathrm{Fe}(Z=26)$ is only about 19.6.

The starting point of quenching correction is the well known Birks law [8]:

$$
\frac{d L}{d x}=\frac{S \frac{d E}{d x}}{1+k B \frac{d E}{d x}},
$$

where $L$ is the light yield, and $k B$ is the quenching parameter. If the charged particles pass through the PSD vertically, we can get the following formula:

$$
\frac{L}{d}=\frac{S E / d}{1+k B E / d}
$$

For DAMPE, the thickness of PSD bar is $d=1$ centimeter. For simplicity, we will omit it hereafter, that is:

$$
L=\frac{S E}{1+k B E} .
$$

For high energy cosmic rays, the most probable energy deposition per unit path length is proportional to the square of their charge numbers: $E=a Z^{2}$. Geant4 simulation shows that $a=2$ $\mathrm{MeV} / \mathrm{cm}$ for a PSD bar. Then Eq. (2.4) becomes

$$
L=\frac{a S Z^{2}}{1+a k B Z^{2}}
$$

As mentioned above, the quenching effect will make the light yield $L$ to deviate from the square of charge number for heavy nuclei. But, in event reconstruction, the relationship between the light yield and the energy deposition is determined by protons. Therefore, for heavy nuclei the energy deposition derived from the light yield is smaller than the real one. Let us call it the apparent energy deposition $\left(E^{*}\right)$ :

$$
L=b E^{*}=a b Z^{* 2} .
$$

Correspondingly, $Z^{*}$ is called the apparent charge number. The coefficient $b$ can be obtained by fitting the correlation function between ADC value and the energy deposition of proton MIP events. By combining Eqs.(2.5) and (2.6) we can get the relationship between the apparent charge number and the real one:

$$
Z^{2}=\frac{Z^{* 2}}{S / b-a k B Z^{* 2}}
$$

However, the results show that this formula cannot reproduce the quenching behavior of PSD very well. One convenient way is to modify the Birks law slightly, like other authors [9] did. In this work, a linear term is introduced into the denominator of the right side of Eq.(2.7):

$$
Z^{2}=\frac{Z^{* 2}}{S / b+c Z^{*}-a k B Z^{* 2}}=\frac{Z^{* 2}}{p_{0}+p_{1} Z^{*}+p_{2} Z^{* 2}} .
$$




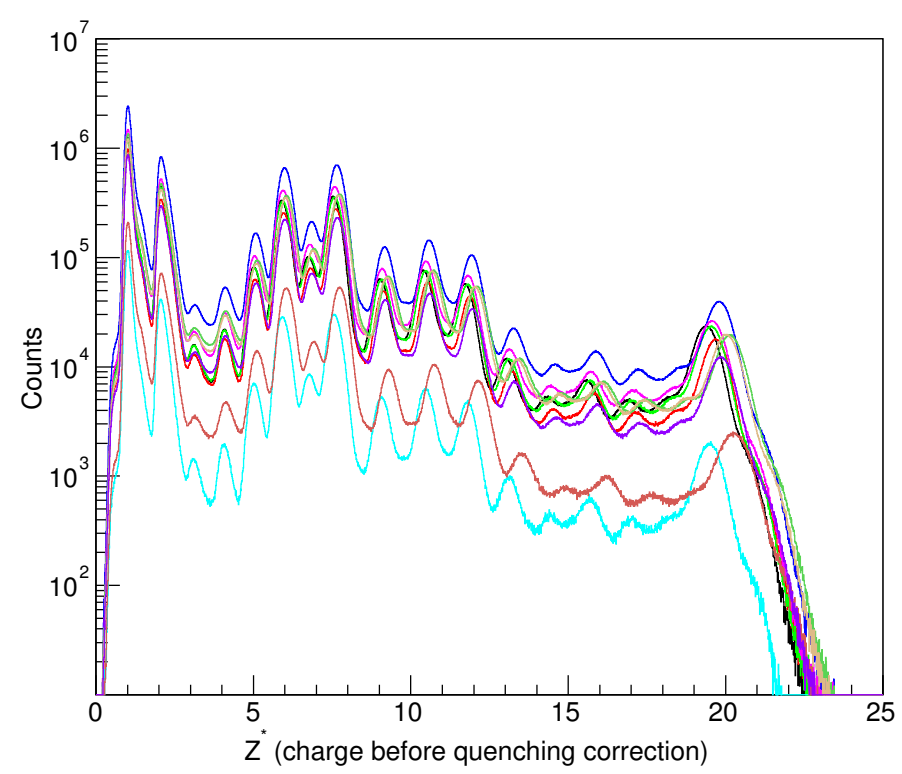

Figure 3: 10 groups of charge spectra by 164 PMTs before quenching and equalization correction. For each group, the peak values of high abundant nuclei are used to fit the quenching function with Eq.(2.8).

It is found that this formula can describe the quenching effect very well.

Although the equalization and quenching corrections are independent one to each other, it is more convenient to make both at the same time. It is because the real charge number of cosmic ray nuclei provides us a natural scale. In fact, we find that the charge spectra obtained by all 164 PMTs can be classified as 10 groups. Here it should be noted that the equalization and quenching corrections are performed after the light attenuation correction. For each group the peaks of charge spectra measured by every PMT are overlapping. The charge spectra for the 10 groups of PMTs are shown in Fig.3. Since the numbers of MPTs in these 10 groups are different, the event number of these groups also varies. The parameters $p_{0}, p_{1}$, and $p_{2}$ in Eq. (2.8) can be obtained by fitting the peak positions of elements for each group.

\section{Charge Measurement Results}

After the above corrections, the charge spectrum detected by the whole PSD is obtained. In this paper we report the results of charge spectrum based on three years of on-orbit data (from 2016-01-01 to 2018-12-31). The results are shown in Fig.4. From this figure we can see that the peaks of most elements from $\mathrm{H}$ to $\mathrm{Ni}$ can be clearly identified. The charge distributions of $\mathrm{H}$ and He can be described by the Gaussian convolved with Landau distribution functions, and the charge distributions of heavier ones can be described well by Gaussian functions. By fitting the whole charge spectrum one can get the resolution of each element. The resolutions for some abundant elements are shown in Table 1. For $\mathrm{H}$ and $\mathrm{He}$, the values of full width at half maximum (FWHM) are 0.137 and 0.238 , respectively. 


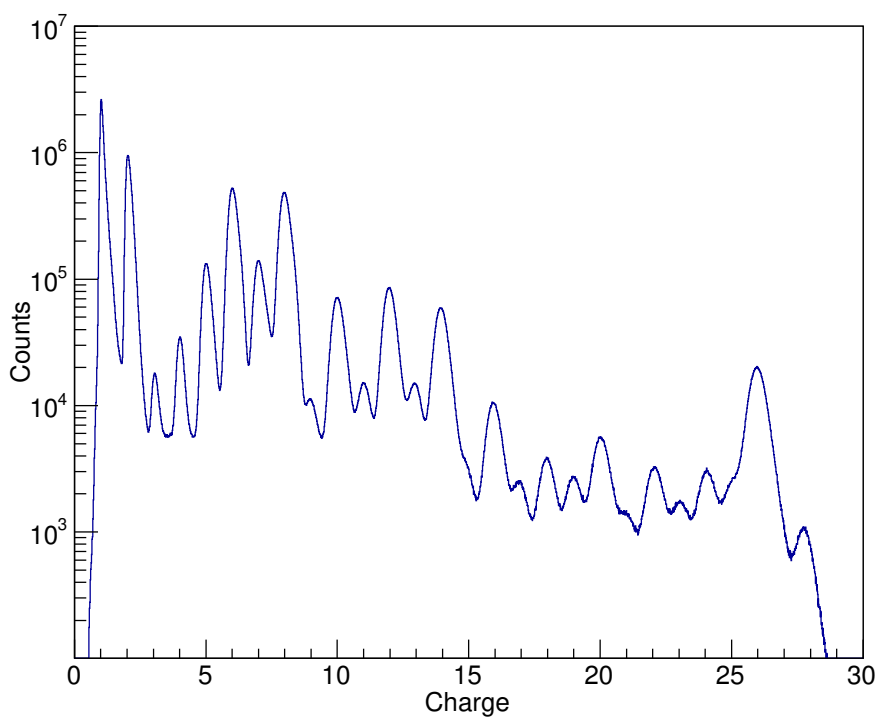

Figure 4: The charge spectrum obtained after making the alignment, light attenuation, equalization and quenching corrections. Three years of on-orbit data are used (from 2016-01-01 to 2018-12-31).

Table 1: Charge resolution of some abundant elements.

\begin{tabular}{lccccr}
\hline Element & $\sigma_{Z}$ & Element & $\sigma_{Z}$ & Element & $\sigma_{Z}$ \\
\hline $\mathrm{B}$ & 0.17 & $\mathrm{Ne}$ & 0.21 & $\mathrm{~S}$ & 0.25 \\
$\mathrm{C}$ & 0.18 & $\mathrm{Mg}$ & 0.22 & $\mathrm{Ca}$ & 0.29 \\
$\mathrm{O}$ & 0.20 & $\mathrm{Si}$ & 0.25 & $\mathrm{Fe}$ & 0.30 \\
\hline
\end{tabular}

\section{Summary}

In summary, the procedure of charge measurement by DAMPE is described and discussed. The basic idea is that the PSD is regarded as a four-layer detector, one PSD bar can measure a particle twice. Hence, PSD can detect the charge of a particle at least 4 times and at most 8 times, and the arithmetic mean value is calculated as the best estimation of its charge. The main steps of charge reconstruction include track finding and four corrections: alignment correction, light attenuation correction, quenching and equalization correction. It is shown that, after these corrections, the PSD of DAMPE can measure cosmic ray nuclei from $\mathrm{H}$ to $\mathrm{Ni}$ with quite high resolution.

\section{References}

[1] J. Chang, Chin. J. Spac. Sci. 34 (2014) 550.

[2] G. Ambrosi et al. (DAMPE collaboration), Nature 552 (2017) 63.

[3] J. Chang et al., Astropart. Phys. 95 (2017) 6.

[4] Y. H. Yu et al., Astropart. Phys. 94 (2017) 1.

[5] T. K. Dong et al., Astropart. Phys. 105 (2019) 31. 
[6] P. X. Ma et al., RAA 19 (2019) 82.

[7] G. Ambrosi et al. (DAMPE collaboration), Astropart. Phys. 106 (2019) 18.

[8] J. B. Birks, Proc. Phys. Soc. A 64 (1951) 874.

[9] R. L. Craun and D. L. Smith, Nucl. Instrum. Meth. 80 (1970) 239. 